normal circumstances, the oxygenation of the tissues is represented by $x-7$. Now if $x$ is diminished by ligature of the main vessel, 7 can also be diminished by ligature of the vein, and by letting the blood drain away more slowly it is more fully deprived of its oxygen. Whether this explanation is true or not is another matter.

To many of the first class for whom the book is intended, and whose "house" days are becoming a bit distant, a little more detail about oxygen therapy would be a help, as this is "new since their day."

The instructions for the after-care of a colostomy, based upon Gabriel's teachings, are clear, and should prove of great help to doctors in advising their patients. It may be pointed out that, for a patient in active work, the morning routine may be too time-consuming. In such cases the colostomy toilet can be performed at night, after work, when there is no hurry, and many patients find this more convenient.

To prevent mistake, the term $5 \%$ glucose solution for intravenous use should be amplified by stating that it is $5 \%$ glucose in distilled water, as a commonly employed solution is $5 \%$ glucose in physiological saline, which would defeat the object which the author rightly desire?. The use of silver nitrate for testing the urip a cases of vomiting is new to me, but sounds an easy and useful indication for the administration of sodium chloride.

In dealing with burns, those most experienced in this work oppose the tanning of the face and hands, yet this is recommended in this work. Some detail of sulphonamide pastes and envelope jaconet dressings would be an advantage, and one would suggest that the author should on his return to this country see the coloured films of this work prepared by Vaughan Hudson at the Middlesex Hospital.

The after-treatment of fractures should prove very helpful, though one wonders if instep, which is defined by the Pocket Oxford Dictionary as "top of foot between toes and ankle" is being confused with arch, when the author states that "sorbo can be applied under the instep."

A number of , books have appeared on "aftertreatment" in recent years, but this seems to cover a wider field than most, and should prove of the greatest use, not only to those for whom it is intended, but to surgical specialists also, who will find useful information and food for thought. It is likely that this work will become a surgical classic, and run into many editions, and it is on account of this belief that one ventures to make the above detailed criticisms and suggestions. One can without hesitation say that no house surgeon should be without this book, and it will prove of immense help to intelligent ward sisters, as well as to those practitioners who undertake the post-operative care of patients, whose sins of omission and commission can do so much to mar a surgeon's work.

\section{TEXT-BOOK OF SURGICAL TREATMENT INCLUDING OPERATIVE SURGERY 2nd Edition}

Edited by C. F. W. Illingworth, M.B., Ch.M., F.R.C.S.Ed. Compiled by eighteen contributors. E. \& S. Livingstone, Edinburgh, 1944. Royal 8vo. pp. 364, 230 illustrations, some in colour. Price 30 .

The fact that a second edition has been issued in r944, though the first edition only appeared in 1943, is perhaps the most satisfying criterion of this work's popularity. The present edition, while fully maintaining the best features of the first edition, has gone far to meet criticisms levelled at certam sections which in the first edition were considered to be below the general high standard of the book. 而 particular, the chapters on amputations and \&n fractures, have been vastly improved. Some account of post-operative treatment of amputations is given and, though one does not want to harp \&n this subject, a few illustrations of exercises a bandaging would be helpful.

One's recollection of one's student days is that the surgeon, as a rule, having amputated a limb, to ${ }^{k}$ no further interest in the progress of the patient. A better conception of a surgeon's duty is given by Langdale-Kelham \& Perkins (Oxford War Manudls -Amputations and Artificial Limbs) who stat "The surgeon's task does not finish with the healing of the wound. It is his further duty (I) to prepare the stump to take an artificial limb, (2) to prepade the patient, so that he can use a limb, and (3) to see that the patient is taught how to use the limb wheh

As Atkins (After Treatment - Blackwell) has pointed out, the work of Simpson and Lister causeg the surgeons whose home had been the ward, to make the theatre their principal residence, but there is now a tendency to return to the ward.

Before leaving the question of amputations, the are two small criticisms. In discussing amputation at the hip, no mention is made of the very real value to the patient of leaving the great trochanter an the head and neck of the femur from, the point of view of a satisfactory artificial limb, if these struc tures can be spared without risk to the health the patient. Secondly, in dealing with amputation in cases of diabetes, the sentence "the operacion should, however, be delayed sufficiently to ensuter adequate preparation" might be expanded a little.

In the chapter on fractures, in dealing abduction of the shoulder, no instruction is give in regard to forward inclination of the arm, and Fig. 50, p. I83, in the chapter on Paralysis an Contraction, shows an arm apparently abducted in the coronal plane. This is entirely opposed Watson-Jones's more usually accepted view that the arm should be carried forward about 30 degrees.

Directions for plaster for a fractured navicular bone could be made more clear by a line drawing.

Despite these perhaps minor criticisms the chan ter on fractures is a great improvement on that in the first edition.

The chapter on burns has been entirely rewritter. by a new vontributor, Mr. Thomas Gibson, who had had special experience in the Burns Unit of Glasgow. Royal Infirmary, and whose excellent contributioi reflects his experience.

Finally, to be in the vogue, illustrations in colou appear. One often wonders if some of these coloug pictures that appear in textbooks are worth the increased cost of production, for often their artistio merit completely outweighs their didactic value? When the question of illustrations is considered, onf would advise any prospective author to studys
Bonney's illustrations in Berkeley \& Bonney'su Gynaecological Surgery, which might well serve as pattern of visual instruction.

One can recommend this second edition even more highly than one recommended the first, not only t\$ those senior students for whom it was intended, but also to many senior practititioners, and even prac $\$$ tising surgeons who may desire to bring their knowledge up to date. 